\title{
A Distributed Paging Mechanism over the X2 Interface for All-Wireless Networks of Small Cells
}

\author{
Jaime Ferragut, Josep Mangues-Bafalluy \\ Mobile Networks Department - Centre Tecnològic de Telecomunicacions de Catalunya (CTTC) \\ Parc Mediterrani de la Tecnologia - Av. Carl Friedrich Gauss, 7 - 08860 Castelldefels - Barcelona, Spain \\ \{jaime.ferragut, josep.mangues\}@cttc.cat
}

\begin{abstract}
In this paper we propose a 3GPP-compliant distributed paging mechanism over the $\mathrm{X} 2$ interface for allwireless Networks of Small Cells (NoS). An all-wireless NoS is a wireless mesh network that provides multi-hop connectivity amongst small cells, as well as access to functional entities in the mobile operator's core network. The standard 3GPP paging procedure has been designed with traditional macrocell scenarios in mind. This raises scalability and network congestion issues in large-scale deployments of all-wireless NoS, where paging messages need to traverse a large number of small cells in order to reach the destination mobile terminal. Our proposal aims at reducing the total number of over-the-air paging transmissions and end-to-end paging delay needed to locate a terminal in a large NoS upon arrival of an incoming voice/data connection request. The scope of the proposed scheme is limited to the NoS, thus allowing all standard procedures and interfaces towards the core network to remain untouched. In addition, new network procedures and protocol messages have been designed to ensure a seamless integration with the latest release of 3GPP Technical Specifications. Simulation results show that our scheme outperforms the standard 3GPP paging mechanism in terms of total number of over-the-air paging transmissions, number of wireless channel accesses per byte, and end-to-end paging delay.
\end{abstract}

Keywords: Long Term Evolution; Network of Small Cells; Location Management; Paging; S1-AP protocol; X2-AP Protocol.

\section{INTRODUCTION}

LTE small cells (also referred to as HeNBs) are gaining momentum as an efficient and cost-effective solution to improve cellular coverage and boost network capacity in home environments. A novel deployment approach, referred to as Networks of Small Cells (NoS), has been studied in the context of the European project BeFEMTO [1] as a solution for the provision of cellular services in large-scale scenarios, such as corporate premises, shopping malls, sports venues, and dense urban areas. In a NoS, small cells collaborate with each other in a self-organizing fashion to optimize network performance and minimize resource consumption. However, operators may not always be able to provide each small cell with a wired backhaul link towards the core network, especially in locations with limited access (e.g., urban furniture). In these scenarios, an all-wireless NoS is as a flexible and cost-effective solution to improve cellular coverage and network capacity. One of the most critical issues is traffic management over the wireless multi-hop backbone. This is particularly challenging for location signaling traffic (i.e., finding a mobile terminal in the network), given the high volume of control messages generated by frequent handovers and cell reselections.
Standard 3GPP location management mechanisms comprise two main building blocks, namely the Tracking Area Update (TAU) and the paging procedures [2]. The goal of the TAU procedure is to enable the Mobility Management Entity (MME) in the core network to track the approximate location of a mobile terminal in the Radio Access Network (RAN). On the other hand, the core network utilizes the paging procedure to notify a User Equipment (UE) of an incoming voice or data connection request. Since 3GPP Technical Specifications define these procedures in the context of traditional macrocell scenarios, their performance and scalability in all-wireless networks of small cells is not optimal. Thus, NoS require custom Tracking Area Update and paging procedures that can track and page UEs efficiently whilst mitigating signaling traffic congestion over the wireless multi-hop backbone.

In this paper we propose a 3GPP-compliant distributed paging mechanism over the X2 interface for all-wireless NoS. Our scheme splits the standard 3GPP paging procedure into two stages in order to reduce the total number of over-the-air (OTA) transmissions in the wireless multi-hop backbone and the end-to-end paging delay. In particular, the proposed scheme sends a single unicast S1-AP Paging message from the Mobility Management Entity (MME) to a selected small cell in the destination Tracking Area (TA). Upon reception of this message, the selected HeNB initiates a local paging procedure via the X2 interface until the destination UE has been paged. In order to avoid unnecessary flooding over the wireless multihop backbone, the MME computes a bijective paging tree for the destination Tracking Area and encapsulates it in the S1-AP and X2-AP message payloads. This is particularly useful for large Tracking Areas, where the number of small cells has an impact on scalability and network performance. Simulation results show that the distributed paging mechanism outperforms the industry standard (i.e., the 3GPP paging mechanism) in terms of total number of OTA transmissions, average end-to-end paging delay, and number of wireless channel accesses (MAC accesses) per byte.

The remainder of this paper is organized as follows. Section II provides some technical background on location management procedures in LTE networks. Previous research work is presented in Section III, followed by a detailed description of the distributed paging mechanism in Section IV. Section V discusses the simulation and performance evaluation of the proposed scheme as compared with the 3GPP standard paging mechanism. Finally, Section VI concludes the paper.

This work has been partially supported by Spanish grants TEC201129700-C02-01 and FPU AP2009-5000, and EU grant ICT-4-248523. 


\section{LocAtion MANAGEMENT PROCEDURES IN LTE NETWORKS}

From the point of view of Radio Resource Control (RRC), an LTE UE can operate in either idle or connected mode [3]. In idle mode, there are no dedicated radio resources between the UE and the network. However, the UE can still receive and decode critical system-level information by listening to broadcast and downlink shared channels. In connected mode, the UE establishes dedicated radio resources with the network as a result of an ongoing voice call or data connection.

The term location management refers to the set of network protocols and procedures used to determine and update the location of a UE in the Radio Access Network. The following subsections discuss location management procedures in macrocell and NoS scenarios, respectively.

TABLE I. LIST OF 3GPP ACRONYMS

\begin{tabular}{|c|c|}
\hline 3GPP & Third-Generation Partnership Project \\
\hline ASN.1 & Abstract Syntax Notation One \\
\hline E-UTRAN & Evolved UMTS Terrestrial Radio Access Network \\
\hline eNB & Evolved Node B \\
\hline EPC & Evolved Packet Core \\
\hline EPS & Evolved Packet System \\
\hline GTP-C & GPRS Tunnelling Protocol, Control Plane \\
\hline HeNB & Home Evolved Node B \\
\hline IE & Information Element \\
\hline LNGW & Local Network of Small Cells Gateway \\
\hline LTE & Long-Term Evolution \\
\hline MAC & Medium Access Control \\
\hline MME & Mobility Management Entity \\
\hline NoS & Network of Small Cells \\
\hline OTA & Over the Air \\
\hline P-MME & Proxy Mobility Management Entity \\
\hline P-SGW & Proxy Serving Gateway \\
\hline PDN-GW & Packet Data Network Gateway \\
\hline RAN & Radio Access Network \\
\hline RRC & Radio Resource Control \\
\hline S-GW & Serving Gateway \\
\hline S-TMSI & SAE Temporary Mobile Subscriber Identity \\
\hline S1-AP & S1 Application Protocol \\
\hline SAE & System Architecture Evolution \\
\hline TA & Tracking Area \\
\hline TAI & Tracking Area Identity \\
\hline TAU & Tracking Area Update \\
\hline UE & User Equipment \\
\hline $\mathbf{X} 2-\mathbf{A P}$ & X2 Application Protocol \\
\hline
\end{tabular}

\section{A. Location Management in Macrocell Networks}

In idle mode, the location of a UE in the Radio Access Network is known on a Tracking Area granularity [2]. A TA is a group of neighboring cells under the control of a Mobility
Management Entity in the core network, or Evolved Packet Core (EPC), in 3GPP terminology. In order to keep its location updated in the MME, a UE performs a Tracking Area Update every time it enters a new TA. When the network needs to notify a UE in idle mode of an incoming voice or data connection, the MME sends an S1-AP Paging message to each cell in the UE's current Tracking Area. Upon receipt of the S1AP Paging message, each cell sends a paging indication over the LTE radio interface. When the UE receives a paging indication from its serving cell, it initiates a Service Request procedure, i.e., it establishes a set of dedicated resources with the RAN and the core network, thus effectively switching from idle to connected mode.

\section{B. Location Management in Networks of Small Cells}

In order to support local mobility and traffic management services, a new functional entity called Local Network of Small Cells Gateway (LNGW) needs to be deployed in the NoS architecture [1]. From the logical point of view, a LNGW acts as a network manager for local mobility, traffic management, access control, authentication, power management, and fault management. LNGWs enable scalable NoS deployments by keeping user- and control-plane traffic within the NoS as much as possible. In addition, LNGWs perform control-plane traffic consolidation towards the EPC similarly to standard HeNB gateways [3]. Thus, all standard 3GPP procedures between the LNGW and the core network remain untouched.

From the functional point of view, a LNGW comprises two entities, namely the Proxy Mobility Management Entity (PMME) and the Proxy Serving Gateway (P-SGW). These building blocks act on behalf of the corresponding core network entities (MME and Serving Gateway (S-GW)) to support mobility and data traffic management in the NoS, respectively. In summary, the P-MME is in charge of location management procedures in the NoS in the same way the MME is in traditional macrocell deployments. For a detailed description of the 3GPP network architecture and functional entities, the reader is referred to [2].

\section{RELATED WORK}

Previous research work on user-tracking mechanisms in cellular networks can be found in the literature [4]-[8]. However, most of these proposals address the issue of location management strictly as an optimization problem and, in general, do not consider the impact of their implementations in commercial networks (e.g., peaks of spurious signaling traffic may flood the network during global Tracking Area reconfigurations). In addition, some of the previous location management schemes do not comply with 3GPP Technical Specifications, which compromises their implementation in commercial networks. Instead, our proposal is fully-standard compliant, as it is based on a new protocol message and Information Element (IE) that can be introduced as a vendorspecific extension to existing X2-AP and S1-AP software protocol stacks. In addition, the scope of the proposed modifications is strictly restricted to the network of small cells.

Analogously, there is a substantial amount of research work on paging mechanisms for cellular networks [9]-[14]. The authors in [9] propose a taxonomy based on the way mobility management entities page the cells in the destination Tracking Area (i.e., cell flooding versus selective paging strategies in order to minimize location signaling traffic in the network). These strategies comprise sequential paging [10], selective paging [11], blanket paging [12], velocity paging [13], and pipeline paging [14]. 
The most common paging mechanism in cellular networks is blanket paging due to its simplicity and effectiveness. In blanket paging schemes, the core network sends paging messages to each cell in the destination Tracking Area upon reception of an incoming voice call or data connection request. Blanket paging generates a substantial amount of location signaling traffic in the RAN, depending on the paging arrival rate and the number of cells in each TA. Consequently, these mechanisms do not perform well in all-wireless networks of small cells.

\section{Distributed Paging Mechanism OVer the X2 INTERFACE}

This section describes the distributed paging mechanism in detail, along with the corresponding S1-AP and X2-AP messages involved:

1) Upon arrival of a voice or data connection request, the P-MME sends a single unicast S1-AP Paging message to a selected HeNB in the destination TA. The selected HeNB can be chosen based on different criteria (minimum distance to the P-MME, route optimization based on minimum traffic congestion, maximum number of one-hop neighbors in the vicinity of the selected HeNB, etc.) In the performance evaluation of the distributed paging mechanism (Section V), we have determined the selected HeNB based on minimum distance to the P-MME.

2) In order to avoid unnecessary paging broadcasts in the destination Tracking Area, the P-MME computes a bijective paging tree prior to sending the unicast S1-AP Paging message to the selected HeNB. The paging tree contains a hierarchical paging sequence with the identities of all HeNBs that will be paged in the destination TA, along with the corresponding HeNBs in charge of paging them. Essentially, the paging tree partitions the destination TA in disjoint branches such that each $\mathrm{HeNB}$ is paged only once.

3) Once computed, the P-MME serializes the paging tree into a Prüfer sequence and encapsulates it in the S1-AP Paging message payload. Prüfer sequences are an efficient mechanism for bijective tree encoding and decoding, as they minimize the number of entries needed to serialize a tree and require very low computational power. For further information on Prüfer sequences, the reader is referred to [15].

4) After receiving the unicast S1-AP Paging message from the P-MME, the selected HeNB sends a paging notification over the LTE radio interface. In parallel, it starts decoding the Prüfer sequence in the S1-AP Paging payload in order to reconstruct the paging tree. Once finished, the HeNB scans the paging tree, extracts the subtrees corresponding to each one-hop neighbor, and computes their corresponding Prüfer sequences. Finally, the HeNB encapsulates these sequences into separate X2-AP Paging messages and sends them to its adjacent HeNBs through the logical $\mathrm{X} 2$ interface.

5) Each neighboring HeNB that receives an X2-AP Paging message over the $\mathrm{X} 2$ interface repeats step 4) until the incoming paging tree is empty.

Figure 1 shows the operation of the standard and distributed paging mechanisms in an all-wireless network of small cells scenario.

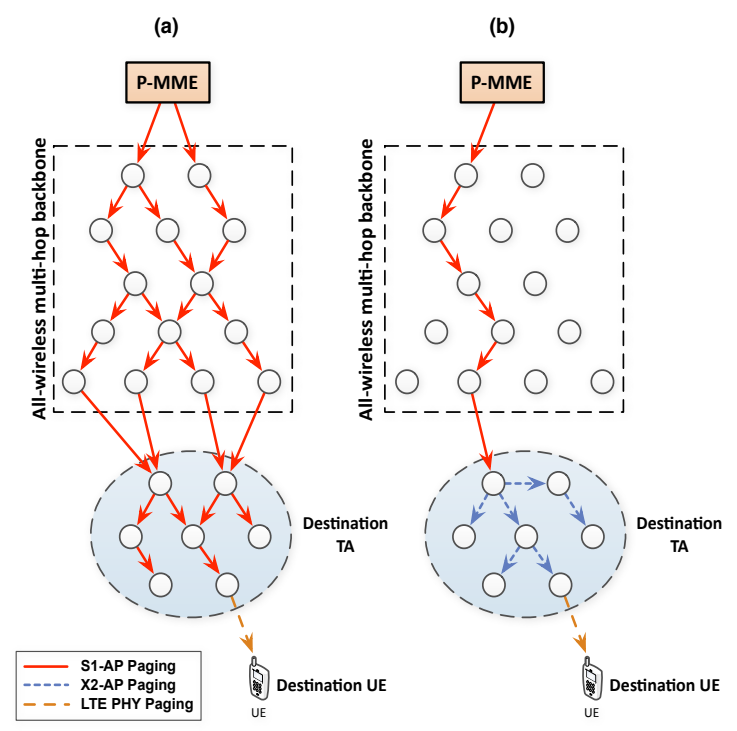

Figure 1. Operation of the standard (a) and distributed (b) paging mechanisms in an all-wireless network of small cells

Figure 2 shows the message sequence chart between functional entities in the distributed paging mechanism. A detailed description of each step is provided below:

1) The Packet Data Network Gateway (PDN-GW) in the core network sends an incoming data packet to the S$\mathrm{GW}$ over the S5/S8 bearer. This indicates that a voice call or data connection needs to be established with a UE in the cellular network.

2) The S-GW forwards the incoming packet to the PSGW in the LNGW over a previously established S1 bearer. For the sake of simplicity, we have assumed that the S-GW entity knows that the UE is currently camped on the network of small cells, although the exact location of the serving HeNB is not known yet.

3) The P-SGW sends a GTP-C Downlink Data Notification message to the P-MME. The purpose of this message is to notify the P-MME that incoming data is ready to be sent to the destination UE.

4) The P-MME obtains the destination TA from the EPS bearer ID field in the GTP-C Downlink Data Notification message and builds a paging tree with the selected HeNB as the root node. All HeNBs in the destination TA are included in the paging tree.

5) The P-MME encodes the paging tree into a Prüfer sequence and encapsulates it in a unicast S1-AP Paging message. This message is sent to the selected HeNB $\left(\mathrm{HeNB}_{1}\right)$, along with the UE SAE Temporary Mobile Subscriber Identity (S-TMSI) and the Tracking Area Identity (TAI).

6) The selected HeNB receives the S1-AP Paging message from the P-MME and sends a paging notification over the LTE radio interface. In parallel, it reconstructs the paging tree and extracts the subtrees rooted at each one of its children nodes (i.e., one-hop neighboring HeNBs in the NoS). Then, each subtree is serialized into a Prüfer sequence and encapsulated into a separate X2-AP Paging message (one for each child HeNB). This is done to reduce the size of the X2-AP Paging payload as paging messages are propagated throughout the wireless multi-hop backbone. 


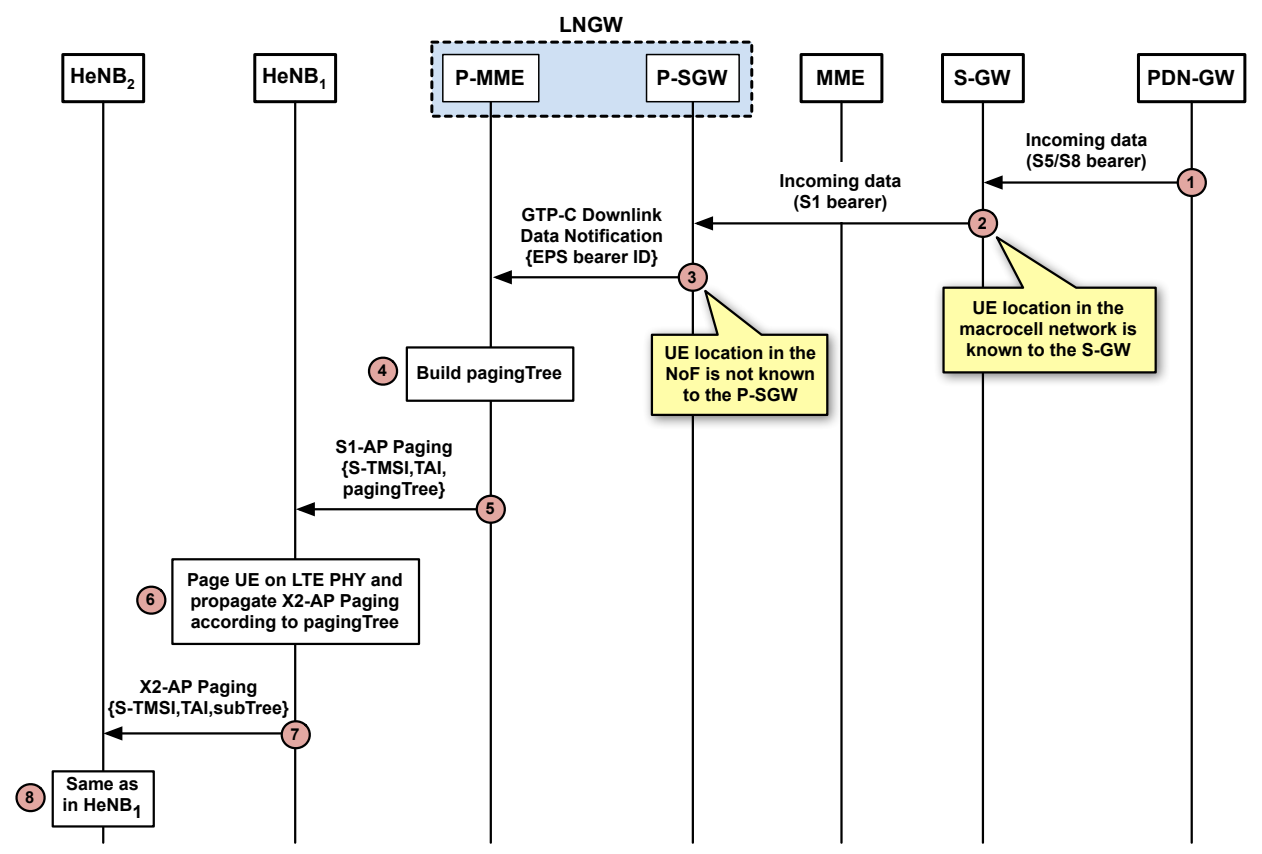

Figure 2. Message sequence chart of the distributed paging mechanism

7) The selected HeNB sends an X2-AP Paging message to each of its one-hop neighboring HeNBs, along with the paging subtree, the UE SAE Temporary Mobile Subscriber Identity (S-TMSI), and the Tracking Area Identity (TAI).

8) Upon reception of an X2-AP Paging message, a neighboring HeNB repeats step 6) until an empty paging tree is received.

In order to encapsulate the Prüfer sequence that contains the paging tree/subtrees, we define a new Information Element in the 3GPP S1-AP and X2-AP protocol specifications. Figure 3 shows the definition of the new PagingTree Information Element in Abstract Syntax Notation One (ASN.1).

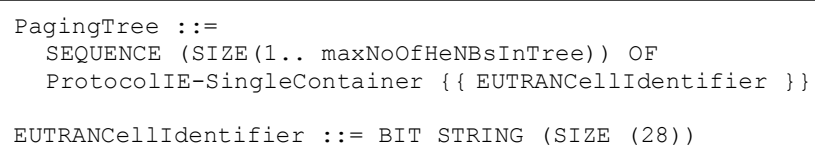

Figure 3. Definition of the PagingTree Information Element in ASN.1

In terms of formal protocol specification, a paging tree is a finite sequence of E-UTRAN Cell Identifiers, i.e., a standard 28-bit string used to identify $(\mathrm{H}) \mathrm{eNBs}$ in the cellular network. In order to ensure full-compliance with 3GPP Technical Specifications, the structure of the PagingTree IE is analogous to that of Tracking Area Lists, as defined in [16].

\section{PERFORMANCE EVALUATION}

In this section we describe the performance evaluation of the distributed paging mechanism as compared with the standard 3GPP paging mechanism. The first subsection defines the evaluation metrics, while the second subsection describes the NoS scenario and the simulation methodology. Finally, the third subsection discusses the simulation results.

\section{A. Evaluation Metrics}

Number of OTA paging transmissions $\left(N_{t x, T o T}\right)$ : this is the total number of S1-AP and X2-AP Paging transmissions over the wireless multi-hop backbone needed to complete a paging procedure. This metric includes MAC retransmissions caused by frame collisions and losses in the wireless channel. Thus,

$$
N_{t x, T O T}=\left\{\begin{aligned}
N_{t x, S 1 A P}, & \text { standard mechanism } \\
\left(N_{t x, S 1 A P}+N_{t x, X 2 A P}\right), & \text { distributed mechanism }
\end{aligned}\right.
$$

Paging payload $\left(\boldsymbol{P}_{\text {paging }}\right)$ : this metric evaluates the total number of S1-AP and X2-AP payload bytes transmitted over the wireless multi-hop backbone during a paging procedure. This metric captures the impact of the PagingTree IE on the S1-AP and X2-AP Paging messages payload.

$$
P_{\text {paging }}=\left\{\begin{aligned}
P_{S 1 A P}, & \text { standard mechanism } \\
\left(P_{S 1 A P}+P_{X 2 A P}\right), & \text { distributed mechanism }
\end{aligned}\right.
$$

Number of MAC accesses per paging payload byte: this is the total number of wireless channel accesses per (S1-AP + $\mathrm{X} 2-\mathrm{AP})$ payload byte, including frame retransmissions. This metric has implications in energy efficiency and end-to-end paging delay, as each MAC access involves RF power consumption, inter-frame waiting periods, and potential delays due to contention mechanisms and retransmissions.

Average end-to-end paging delay: this metric evaluates the average time needed to complete a successful paging operation. It is defined as the time elapsed between the arrival of the GTP-C Downlink Data Notification message to the P-MME and the reception of the last S1-AP/X2-AP Paging message in the destination Tracking Area.

\section{B. Simulation Scenario and Methodology}

In order to evaluate the distributed paging mechanism we use the ns-3 network simulator. We consider a fully-meshed, (6x6)-node wireless multi-hop network with $35 \mathrm{HeNBs}$ and a single P-MME located in the top-right corner of the Radio Access Network. Assuming a maximum coverage radius of 200 meters for commercial small cell equipment [16], this scenario describes a generalization of a simple dense urban deployment of approximately $5 \mathrm{~km}^{2}$, such as a commercial area or business district. In order to evaluate the scalability of the proposed scheme, more complex scenarios with larger number of small cells, multiple Tracking Area sizes, and different PMME locations have also been considered, as described later in this Section. In terms of mesh connectivity, each HeNB communicates with its one-hop neighbors via the logical X2 
interface. This interface is mapped to a physical $802.11 \mathrm{~g}$ link between neighboring HeNBs. There are 4 Tracking Areas in total, each one comprising a subset of $3 \times 3$ HeNBs. UEs move throughout the NoS according to a Gauss-Markov mobility model with speeds that range from 5 to $50 \mathrm{~km} / \mathrm{h}$, thus covering both pedestrian and vehicular users. GTP-C Downlink Data Notification messages arrive to the P-MME following a Poisson process with arrival rate $\lambda \in[0.1,20]$ pagings $/ \mathrm{h}$. In order to average the simulation results, we run 100 repetitions for each paging scheme (standard vs. distributed). The duration of each repetition is 100000 seconds.

Table II summarizes the simulation parameters for the performance evaluation of the distributed paging mechanism.

TABLE II. SIMULATION PARAMETERS FOR PERFORMACE EVALUATION

\begin{tabular}{|c|c|c|}
\hline Name & Description & Value \\
\hline$\lambda$ & Paging arrival rate $[$ pagings $/ h]$ & {$[0.1-20]$} \\
\hline$N$ & Total number of nodes & $\begin{array}{c}35 \mathrm{HeNBs} \\
1 \mathrm{P}-\mathrm{MME}\end{array}$ \\
\hline$N_{\text {femtos, } T A}$ & Number of HeNBs per TA & 9 \\
\hline$v$ & UE speed $[\mathrm{km} / \mathrm{h}]$ & $5-50$ \\
\hline$N_{r}$ & Number of repetitions & 100 \\
\hline$T$ & Repetition duration $[s]$ & 100000 \\
\hline
\end{tabular}

\section{Simulation Results}

Figure 4(a) shows the total number of OTA paging transmissions (S1-AP and X2-AP) over the wireless multi-hop backbone in the standard and distributed paging mechanisms. As seen in the figure, the number of OTA transmissions is consistently lower in the distributed mechanism for all values of $\lambda$. In addition, the variance of the number of transmissions is also lower in the distributed scheme, and remains bounded as $\lambda$ increases. This is a consequence of the distributed paging algorithm. By sending a single S1-AP Paging message to the selected HeNB followed by one-hop X2-AP Paging messages in the destination Tracking Area, the total number of OTA paging transmissions needed to reach all HeNBs is reduced. Furthermore, this has a positive effect on network congestion, thus mitigating channel contention, collisions, and frame retransmissions.

Figure 4(b) shows the impact of the PagingTree Information Element on the S1-AP and X2-AP Paging payload. As depicted in the figure, the total number of OTA payload bytes in the distributed mechanism is higher than in the standard mechanism. This is due to the encapsulation of the Prüfer sequences containing the paging tree (and subtrees) in the S1-AP and X2-AP payloads, respectively. In both schemes, the variance of the number of OTA payload bytes is caused by frame retransmissions. However, the impact of these retransmissions on each mechanism differs. In the standard 3GPP paging scheme, only S1-AP Paging messages can be retransmitted. These messages always contain a single $E$ UTRAN Cell Identifier ( 28 bits) that determine the target HeNB in the destination Tracking Area. As far as the distributed mechanism is concerned, both S1-AP and X2-AP Paging messages can be retransmitted. The S1-AP Paging payload is fixed, since it always contains the entire paging tree. However, the X2-AP Paging payload is variable, as it depends on the size of the extracted subtree. This explains why the variance of the number of OTA payload bytes is higher in the distributed mechanism than in the standard mechanism.

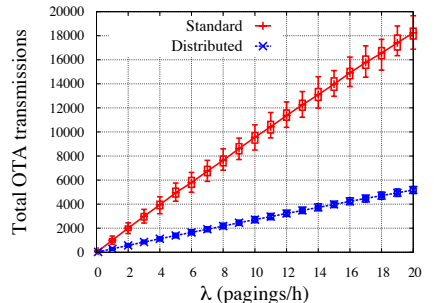

(a)

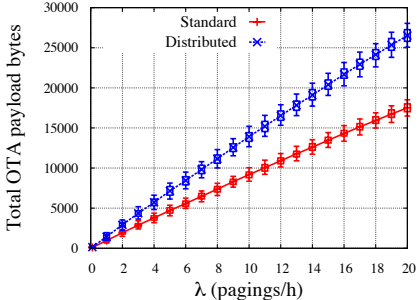

(b)
Figure 4. Total number of over-the-air paging transmissions (a) and total number of over-the-air paging payload bytes (b)

Figure 5 shows the ratio between the number of MAC accesses (including retransmissions) and the S1-AP plus X2AP payload bytes needed to complete a paging operation. We have defined two scenarios in order to evaluate this metric under different network conditions. In the first setup, we have reduced network congestion in the wireless multi-hop backbone by pre-populating the Address Resolution Protocol (ARP) tables for all HeNBs in the network of small cells. We refer to this setup as the background traffic-free scenario. In the second setup, we have enabled ARP traffic between HeNBs to increase network congestion, thus triggering channel contention, collisions and frame retransmissions. This setup is referred to as the background traffic scenario.

Figure 5(a) shows the number of MAC accesses per payload byte in the background traffic-free scenario. As seen in the figure, our proposal outperforms the standard mechanism for all paging arrival rates. To this extent, the lower number of over-the-air transmissions needed to complete a paging procedure in the distributed mechanism compensates the bigger payload caused by the encapsulation of the paging tree. Analogously, Figure 5(b) shows the number of MAC accesses per payload byte in the background traffic scenario. As seen in the figure, the presence of ARP traffic in the wireless multi-hop backbone impacts the performance of both paging mechanisms. In general, our proposal generates less ARP traffic than the standard mechanism due to the lower number of wireless channel accesses needed to complete a paging operation. Furthermore, it presents a decreasing trend as $\lambda$ increases due to the smaller inter-packet transmission intervals, hence not allowing the ARP caching timers for the various entries to expire. When this happens, no new ARP packets are sent, and so, the number of MAC accesses decreases. Although the standard scheme also benefits from the same effect, this is not enough to compensate for the additional retransmissions generated due to the higher number of MAC accesses and, hence, higher contention. This explains why the standard mechanism shows the opposite trend of that of the proposed approach. In both figures, the higher variance in the standard mechanism for $\lambda \leqslant 6$ pagings/h is caused by the sparse arrival of GTP-C Downlink Data Notification messages during simulation time. This becomes less noticeable as $\lambda$ increases.

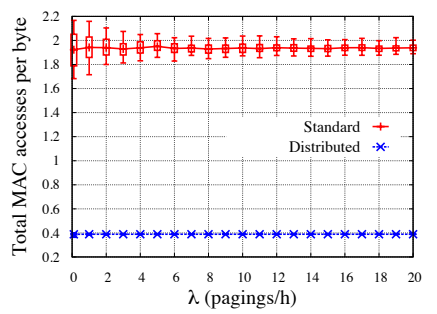

(a)

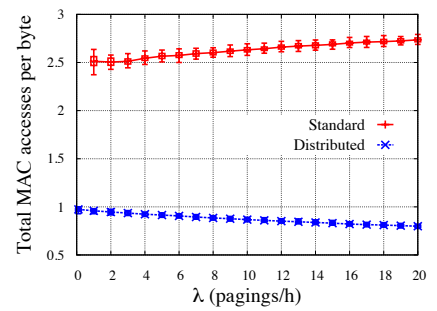

(b)
Figure 5. Total number of MAC accesses per paging payload byte in the background traffic-free (a) and background traffic (b) scenarios 
Figure 6 shows the end-to-end paging delay in the standard and distributed paging mechanisms for both network scenarios. For clarity purposes, only the average values over all simulation repetitions are shown in the figure. As seen in Figure 6(a), the distributed paging mechanism reduces the average end-to-end paging delay by $50 \%$ compared to the standard mechanism in the absence of background ARP traffic. This due to the lower number of multi-hop paging transmissions needed to reach all HeNBs in the destination Tracking Area. In the standard mechanism, each S1-AP Paging message needs to traverse the multi-hop wireless backbone of the network of small cells before reaching its destination HeNB. This involves channel contention, transmission delays, and frame retransmissions, hence increasing the end-to-end paging delay. Instead, in the distributed scheme, the P-MME reduces the number of transmissions (and, therefore, contention and collisions) in the wireless channel by sending a single S1AP Paging message to a selected HeNB in the destination Tracking Area. All subsequent X2-AP Paging messages between neighboring HeNBs in the destination Tracking Area are (by definition) one-hop transmissions. This reduces channel contention, collisions, and frame retransmissions, thus resulting in a lower end-to-end paging delay.

As seen in Figure 6(b), the presence of background ARP traffic in the wireless multi-hop backbone increases the end-toend paging delay for both paging mechanisms. Higher traffic load leads to higher contention in the wireless channel, thus resulting in more collisions and frame retransmissions. In turn, this translates into higher values of end-to-end paging delay. Furthermore, additional delays caused by the ARP mechanism are a key contributing factor to the overall end-to-end paging delay. In general, MAC entries in ARP tables are more likely to expire for lower values of paging arrival rates. Consequently, HeNBs need to re-populate their ARP tables every time a GTP-C Downlink Data Notification message reaches the P-MME, therefore leading to higher values of endto-end paging delays. As $\lambda$ increases, voice and data connection requests reach the P-MME before MAC entries in the ARP tables have expired, which eliminates the need for restarting ARP mechanisms in the network. In accordance with the discussion on Figure 5(b), it is clear the role that MAC accesses play in the end-to-end paging delay, as the trends are the same except for the ripple in the curve of the standard mechanism due to the sparsity of paging arrivals for low values of $\lambda$, as explained above.

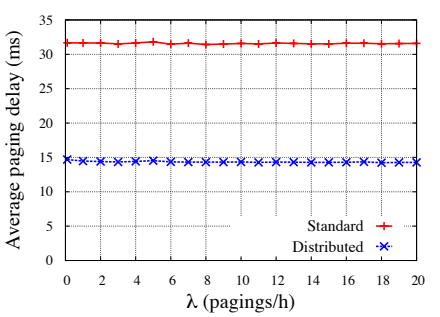

(a)

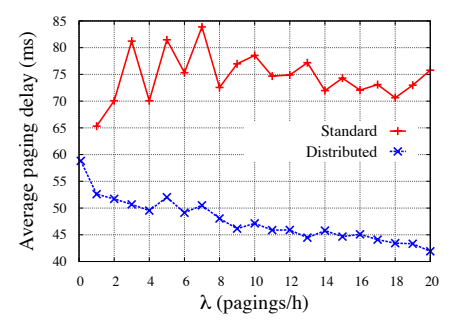

(b)
Figure 6. Average end-to-end paging delay in the background traffic-free (a) and background traffic (b) scenarios

In order to evaluate the scalability of the distributed paging mechanism we define a set of more complex simulation scenarios. In particular, we consider a fully meshed $(24 \times 24)$ node wireless multi-hop network with $575 \mathrm{HeNBs}$ and one PMME located in the center of the network of small cells. As in the previous scenario, each HeNB can only communicate with its one-hop neighbors via a logical X2 interface mapped to a physical 802.11g link. As far as the Tracking Area size is concerned, we consider six different cases, namely 1, 4, 9, 16, 36, and 64 small cells per Tracking Area. UEs move throughout the NoS according to a Gauss-Markov mobility model with speeds that range from 5 to $50 \mathrm{~km} / \mathrm{h}$. GTP-C Downlink Data Notification messages reach the P-MME following a Poisson process with arrival rate $\lambda=10$ pagings $/ \mathrm{h}$. In order to average the simulation results, we run 50 repetitions for each paging scheme (standard vs. distributed). The duration of each repetition is 10000 seconds. These parameters are summarized in Table III.

TABLE III. SIMULATION PARAMETERS FOR SCALABILITY EVALUATION

\begin{tabular}{|c|c|c|}
\hline Name & Description & Value \\
\hline$\lambda$ & Paging arrival rate $[$ pagings $/ h]$ & 10 \\
\hline$N$ & Total number of nodes & $\begin{array}{c}575 \mathrm{HeNBs} \\
1 \mathrm{P}-\mathrm{MME}\end{array}$ \\
\hline$N_{\text {femtos, } T A}$ & Number of HeNBs per TA & $1,4,9,16,36,64$ \\
\hline$v$ & UE speed $[\mathrm{km} / \mathrm{h}]$ & $5-50$ \\
\hline$N_{r}$ & Number of repetitions & 50 \\
\hline$T$ & Repetition duration $[s]$ & 10000 \\
\hline
\end{tabular}

Figure 7 shows the total number of OTA transmissions needed to complete a paging procedure for different Tracking Area sizes. As seen in the figure, the distributed paging mechanism scales better than the 3GPP standard mechanism for large deployments of all-wireless networks of small cells. As the total number of HeNBs increases, each S1-AP Paging message needs to traverse a higher number of hops in the NoS in order to reach its destination. In the standard mechanism, the total number of OTA transmissions in a paging procedure scales by the number of hops of a single S1-AP Paging message times the number of HeNBs in the destination TA. However, in the distributed mechanism, the dominating scaling factor in the total number of OTA transmissions is the number of HeNBs in the destination TA only.

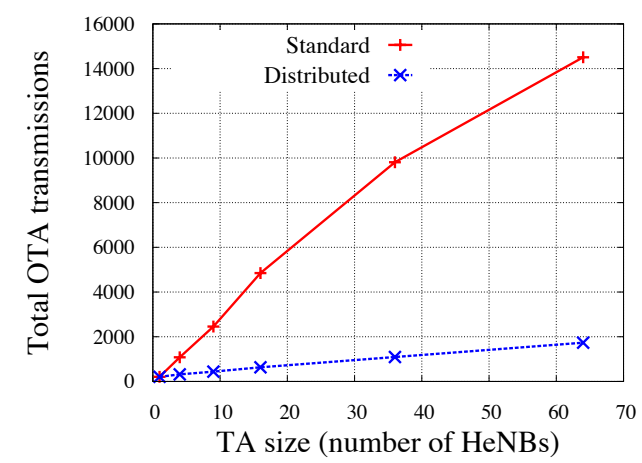

Figure 7. Total number of over-the-air paging transmissions for different Tracking Area sizes

Figure 8 shows the total number of OTA payload bytes in S1-AP and X2-AP Paging messages for different Tracking Area sizes. As expected, the number of payload bytes in the distributed mechanism is higher than in the standard mechanism due to the encapsulation of the paging tree (and subtrees). In the standard scheme, the total number of OTA payload bytes scales by the number of S1-AP Paging messages times the size of a single E-UTRAN Cell Identifier. However, in the distributed scheme, the total number of OTA payload bytes needed to complete a paging operation scales by the size of the paging tree/subtrees and the total number of S1-AP and X2-AP Paging messages. 


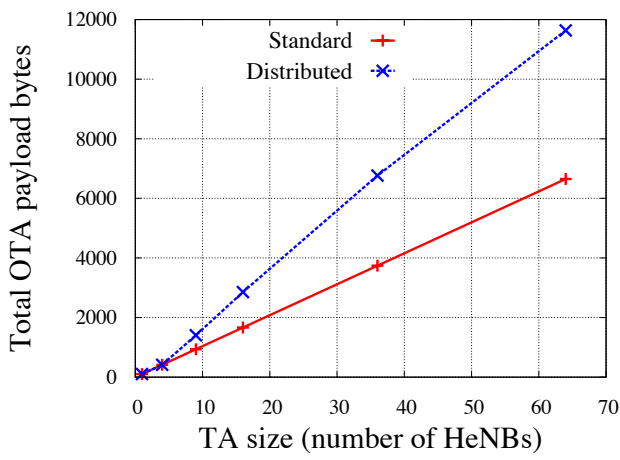

Figure 8. Total number of over-the-air paging payload bytes for different Tracking Area sizes

Figure 9 shows the total number of MAC accesses per payload byte for different Tracking Area sizes. As seen in the figure, the distributed paging mechanism outperforms the standard mechanism in terms of number of wireless channel accesses per byte. This is achieved by packing a higher number of bytes (i.e., the paging tree and subtrees) in fewer OTA transmissions. The positive impact of the paging tree/subtrees in the number of total MAC accesses per byte becomes much more noticeable as the number of HeNBs in the destination Tracking Area increases.

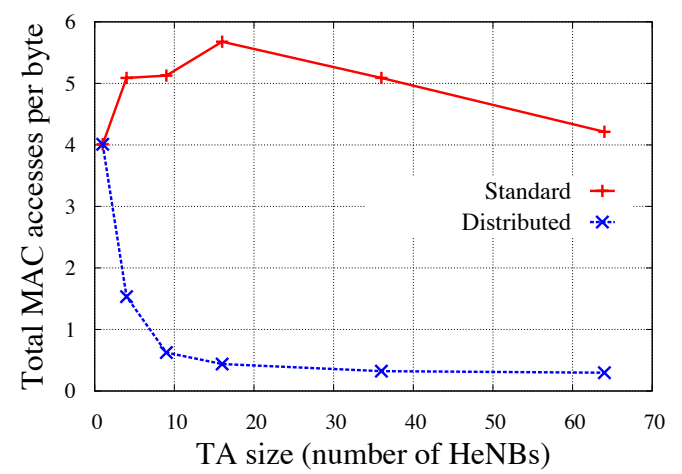

Figure 9. Total number of MAC accesses per paging payload byte for different Tracking Area sizes

Figure 10 shows the average end-to-end paging delay for different Tracking Area sizes. As shown previously, channel contention, collisions, and frame retransmissions are the key contributing factors to end-to-end paging delay. Since the distributed paging scheme reduces congestion in the wireless multi-hop backbone, a lower number of S1-AP and X2-AP Paging messages experience channel contention, collisions and frame retransmissions. Consequently, the positive impact of lower network congestion on end-to-end paging delay becomes more noticeable as the size of the destination TA increases.

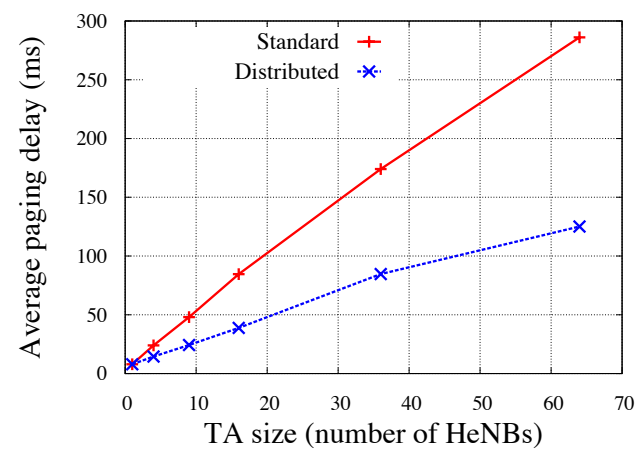

Figure 10. Average end-to-end paging delay for different Tracking Area sizes

\section{CONCLUSIONS}

In this paper we have proposed a 3GPP-compliant distributed paging mechanism over the X2 interface for allwireless networks of small cells. The proposed mechanism is based on a combination of unicast S1-AP paging from the PMME to a selected small cell in the destination Tracking Area, followed by a hierarchical sequence of X2-AP paging operations between neighboring small cells.

We have evaluated the performance of the distributed paging mechanism with the ns-3 network simulator. Our results show that the proposed scheme outperforms the 3GPP paging mechanism in terms of total number of over-the-air paging messages over the wireless multi-hop backbone (including frame retransmissions) and wireless channel accesses (MAC accesses) per byte, whilst keeping the paging payload under control. This has a positive impact on end-to-end paging delay, network congestion, and energy consumption. Finally, the distributed paging scheme has been designed in full compliance with 3GPP Technical Specifications in order to facilitate its implementation in commercial networks.

\section{REFERENCES}

[1] BeFEMTO, Broadband Evolved Femto Networks [Online]. Available: http://www.ict-befemto.eu

[2] 3GPP TS 23.401: "General Packet Radio Service (GPRS) enhancements for Evolved Universal Terrestrial Radio Access Network (E-UTRAN) access, v.8.14.0 (Release 8)", June 2011.

[3] 3GPP TS 36.300: "Evolved Universal Terrestrial Radio Access (EUTRA) and Evolved Universal Terrestrial Radio Access Network (EUTRAN); Overall description; Stage 2, v.11.0.0”, March 2012.

[4] I.F. Akyildiz and W. Wang, "On the estimation of user mobility pattern for location tracking in wireless networks", in IEEE Global Telecommunications Conference (GLOBECOM), pp. 610-614, 2002.

[5] S. M. Razavi and D. Yuan, "Performance improvement of LTE tracking area design: a re-optimization approach," in Proceedings of the 6th ACM international symposium on Mobility management and wireless access, pp. 77-84, 2008.

[6] S. M. Razavi, D. Yuan, F. Gunnarsson, and J. Moe, "Exploiting tracking area list for improving signaling overhead in LTE," in IEEE 71 st Vehicular Technology Conference, pp. 1-5, 2010.

[7] S. M. Razavi, D. Yuan, F. Gunnarsson, and J. Moe, "Dynamic tracking area list configuration and performance evaluation in LTE", in IEEE Globecom Workshops, pp. 49-53, 2010.

[8] Y. Watanabe and M. Yabusaki, "Mobility/traffic adaptive location management", in IEEE 56th Vehicular Technology Conference, pp. 1011-1015, 2002.

[9] P. Das and A. Chandra, "Location management in wireless networks: A survey", in IEEE World Congress on Information and Communication Technologies, pp. 576-580, 2011.

[10] D.J. Lee and D.H. Cho, "Sequential paging schemes considering two classes of paging traffic", in Electronics Letters, vol.35, no.14, pp.1138$1139,1999$.

[11] A. Abutaleb and V. O. K. Li, "Paging strategy optimization in personal communication systems", Wireless Networks, vol. 3, no. 3, pp. 195-204, 1997.

[12] S. Mishra and O.K. Tonguz, "Analysis of intelligent paging in personal communication systems", in Electronics Letters, vol.34, no.1, pp.12-13, 1998.

[13] K. Lam, B. Y. Liang, and C. Zhang, "On using handoff statistics and velocity for location management in cellular wireless networks", The Computer Journal, vol. 48, no. 1, pp. 84-100, 2005.

[14] Y. Xiao, H. Chen, and M. Guizani, "Performance evaluation of pipeline paging under paging delay constraint for wireless systems", in IEEE Transactions on Mobile Computing, vol. 5, no. 1, pp. 64-76, Jan. 2006.

[15] H. Prüfer, "Neuer Beweis eines Satzes über Permutationen". Archiv für Mathematik und Physik, 27, pp. 142-144, 1918.

[16] 3GPP TS 36.413: "Evolved Universal Terrestrial Radio Access Network (E-UTRAN); S1 Application Protocol (S1AP), v.10.5.0”, March 2012.

[17] NETGEAR Femtocell Voice Gateway DVG834GH, datasheet [Online]. Available: http://www.netgear.com 